\title{
Resultative approach to distribution of budget resources of municipalities
}

\author{
Olga Rostova $^{1, *}$, Svetlana Shirokova ${ }^{1}$, and Anastasiia Shmeleva ${ }^{1}$ \\ ${ }^{1}$ Peter the Great St.Petersburg Polytechnic University, Polytechnicheskaya, 29, St. Petersburg, \\ 195251, Russia
}

\begin{abstract}
The article is devoted to the problem of rational allocation of budget resources of municipalities, which is proposed to be solved by an effective approach. The essence of this approach lies in the change of the focus of the budget management process from budget resources management to results management. A mathematical model that can become a basis for making reasonable decisions in process of allocation of budget resources to municipalities is proposed in the article.
\end{abstract}

\section{Introduction}

Limited amount of resources of municipalities, particularly financial resources, determines the need in their rational distribution, systematic monitoring, and development of priority areas of urban financing policy.

Today, there is no generally accepted methodological approach to formation of programmes, plans and principles of budget resources allocation, which can serve as a tool for development and implementation of scientifically based local social and economic policies. Moreover, the procedure and coordination of work on formation of forecast, strategic and planning documents has not been elaborated yet. There is a strong need in a new approach to the role of planned regulation in the process of municipal management and development of new economic and mathematical models and mechanisms for analyzing and forecasting the emerging situation, allocation of budget resources, monitoring their implementation, conducting work on creating and updating the database of statistical reports and planned regulation [1].

The problem of rational distribution of resources was investigated by Gradov A.P., Kuzin B.I., Mednikov M.D., Korobko V.I. [2, 3]. There is a large number of papers and studies devoted to elaboration of economic and mathematical models for development of the regional economy. The research works also represent profound interest $[4,5,6]$. The role of strategic and budget planning in the development of municipalities is revealed in papers of Syatchikhina S.V., Ruchkina A.V., Vasilyeva V.B. [7, 8, 9]. The problem of optimal distribution of a limited amount of budget resources according to the fields of economy is proposed to be considered from the point of conflict management. Classical decision-making mechanisms for distribution of costs, such as egalitarianism and utilitarianism, are used as the basic principles of this theory $[10,11]$.

\footnotetext{
*Corresponding author: o.2908@mail.ru
} 
Limited amount of resources of municipalities determines the need in their rational distribution and constant analysis of the current situation. Consequently, a resultative approach to allocation of budget resources, which is concluded in the change of the focus of budget management process from budget resources management to results management, is proposed $[12,13]$.

\section{Experimental section}

For a more rational distribution process of budget resources of municipalities, an economic and mathematical model was developed to predict the level of development of monitored areas of activity depending on the established priorities and allocated amount of budget.

Consideration of the optimization dynamic model of resources distribution looks the following way:

$$
\left\{\begin{array}{r}
\sum_{i=1}^{n}\left|\frac{\overline{y_{i}}-y_{i}(t+1)}{\overline{y_{i}}}\right| \rightarrow \min _{u(t)} \\
\max _{i=1, n}\left|\frac{\overline{y_{i}}-y_{i}(t+1)}{\overline{y_{i}}}\right| \rightarrow \min _{u(t)} \\
\sum_{i=1}^{n} u_{i}(t)=C(t) \\
y_{i}(t+1)=y_{i}(t)+s_{i}(t) \cdot u_{i}(t)-d_{i}(t) \\
y_{i}(t) \geq 0, s_{i}(t) \geq 0, u_{i}(t) \geq 0 \\
\forall i=\overline{1, n} ; \forall t=\overline{1, T-1}
\end{array}\right.
$$

$C(t)^{-}$is an amount of budget available for investment in the moment $t$;

$t=\overline{1 \ldots T}-$ is a period of forecast;

$i=\overline{\mathbf{1 \ldots n}}$ - is a direction of investment (supposing that the development of each direction is independent);

$y_{i}(t)$ - is a quantitative index of the $\mathrm{i}$-th direction in the moment $\mathrm{t}$;

$y_{i}=y_{i}(T)-$ is an independently determined desired state of i-th direction (according to the future-oriented development plan);

$u_{i}(t)$ - is an amount of budget invested in the i-th direction in the moment $\mathrm{t}$;

$s_{i}(t)$ - is efficiency of investment in the i-th direction (growth of the industry per unit of investment); $\frac{y_{i}(t)}{y_{i}}$ - is a normalized level of the $\mathrm{i}$-th direction;

$d_{i}(t)$ - is an impact of external factors on the state of the i-th direction.

The following values are assumed for the whole forecast horizon: $C(t), s_{i}(t)$

As a result of solving the mathematical programming problem with respect to the variables $\mathrm{u}_{1}, \ldots, \mathrm{u}_{\mathrm{n}}$, at each moment of time we have the optimal distribution of the determined amount of budget $\mathrm{C}(\mathrm{t})$ in the form of the resource allocation vector 
$\left(\mathrm{u}_{1}(\mathrm{t}), \ldots \mathrm{u}_{\mathrm{n}}(\mathrm{t})\right)$, as well as the values of the monitored indicators of economic activity $\mathrm{y}_{\mathrm{i}}(\mathrm{t})$ by years up to the period of $\mathrm{T}$.

The designed model allows to solve the problem of forecasting of the industries' development and optimal allocation of resources for a given period of time with the determined planning interval. In a more general form of the task, the objective functions can be different depending on the priorities defined by the management agent.

\subsection{Resource Allocation Policies}

1. The model of "Equal direction development" is applied in case when the objective is to gradually smooth the disproportions. The policy is analogous to the objective of egalitarianism, however, it is softer, as a certain amount of budget is invested in all areas. There is a pull-up of objects in proportion to their lag. This distribution allows to simultaneously transform all objects from the initial state to the desired one.

The objective function that defines this policy is the following:

$$
f_{\mathrm{O}}(t, u(t))=\sum_{i=1}^{n}\left|u_{i}(t)-\frac{\frac{\overline{y_{i}}-y_{i}(t+1)}{s_{i}(t)}}{\sum_{i=1}^{n} \frac{\overline{y_{i}}-y_{i}(t+1)}{s_{i}(t)}} \cdot C(t)\right| \rightarrow \min
$$

2. The model of "Distribution proportional to the required results" assumes that more budget is allocated to those areas where the deviation from the standard is greater. The objective of the model is to reduce the disproportions in the development of areas. According to its concept, the model is similar to the model of egalitarianism, but it is softer, because a certain amount of budget is allocated to the developed areas.

The objective function that defines this policy is the following:

$$
f_{\mathrm{O}}(t, u(t))=\sum_{i=1}^{n}\left|u_{i}(t)-\frac{\frac{\overline{y_{i}}-y_{i}(t+1)}{\overline{y_{i}}}}{\sum_{i=1}^{n} \frac{\overline{y_{i}}-y_{i}(t+1)}{\overline{y_{i}}}} \cdot C(t)\right| \rightarrow \min
$$

In the numerator, there is a normalized deviation of the i-th direction. In the denominator, there is a sum of the normalized deviations of the entire system. Thus, the coefficient at $C(t)$ shows the share of the normalized deviation of the $\mathrm{i}$-th direction in the sum of all deviations. Although investment is distributed to all areas, for those with a deviation greater, a large amount of budget is allocated.

3. The model of "Distribution proportional to the direction development level". The objective of this policy is to give priority to the fast completion of work and achievement of a benchmark for areas with a high level of development.

The objective function that defines this policy is the following:

$$
f_{\mathrm{O}}(t, u(t))=\sum_{i=1}^{n}\left|u_{i}(t)-\frac{\frac{y_{i}(t+1)}{\overline{y_{i}}}}{\sum_{i=1}^{n} \frac{y_{i}(t+1)}{\overline{y_{i}}}} \cdot C(t)\right| \rightarrow \min
$$


In the numerator, there is a normalized level of the i-th direction. The denominator is the normalized level of the entire system. Thus, the coefficient at $C(t)$ shows the share of the normalized level of the i-th direction in the total normalized level of the system.

4. The model of "Allowable deviations". In the implementation of this model, allowable values of deviations from the benchmark states are specified for certain areas. The areas of allowable deviations, can be as underdeveloped, but of primary importance, as effective with high $\mathrm{s}_{\mathrm{i}}(\mathrm{t})$, but which development is not relevant.

The objective function that defines this policy looks the following way:

$$
\begin{aligned}
& f_{0}(t, u(t))=\sum_{i=1}^{n}\left(\frac{\overline{y_{i}}-y_{i}(t+1)}{\overline{y_{i}}}-p_{i}(t)\right) \rightarrow \mathrm{min} \\
& \frac{\overline{y_{i}}-y_{i}(t+1)}{\overline{y_{i}}} \geq p_{i}(t), \forall i=\overline{1, n}, \forall t=\overline{1, T}
\end{aligned}
$$

$p_{i}(t)$ is a dimensionless quantity indicating the allowable deviation from the benchmark state.

5. The model of "Guaranteed minimum" is applied in case of vital, but underdeveloped areas, when there is a need for their development at least to some certain level. The remaining resources are used to increase overall welfare.

The objective function that defines this policy is the following:

$$
\begin{aligned}
& f_{0}(t, u(t))=\sum_{i=1}^{n}\left(q_{i}(t)-\frac{\overline{y_{i}}-y_{i}(t+1)}{\overline{y_{i}}}\right) \rightarrow \min \\
& \frac{\overline{y_{i}}-y_{i}(t+1)}{\overline{y_{i}}} \leq q_{i}(t), \forall i=\overline{1, n}, \forall t=\overline{1, T}
\end{aligned}
$$

After the allocation of budget, the deviation of the chosen direction from the benchmark should not be greater than the indicated value $q_{i}(t)$, where $q_{i}(t)$ is a dimensionless quantity indicating the maximum possible deviation of the i-th direction.

6. The "Priority setting" model is aimed at the distribution of budget in a manner similar to the "utilitarianism" model, but a criterion of priority is defined by pre-determined

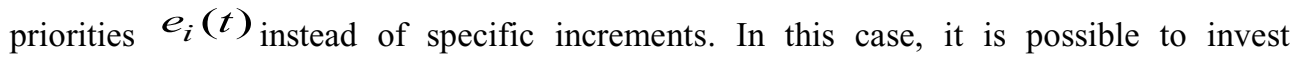
purposefully in the most profitable areas, which development is identified as a priority. $e_{i}(t)$ is a controlling parameter that allows to exert a controlling influence on the allocation of budget for each of the areas.

The objective function that defines this is policy is the following:

$$
\begin{gathered}
f_{0}(t, u(t))=\sum_{i=1}^{n}\left|\frac{\overline{y_{i}}-y_{i}(t)-e_{i}(t) \cdot s_{i}(t) \cdot u_{i}(t)+d_{i}(t)}{\overline{y_{i}}}\right| \rightarrow \min \\
0 \leq e_{i}(t) \forall i=\overline{1, n}, \forall t=\overline{1, T}
\end{gathered}
$$

The mathematical model of optimal allocation of resources was implemented in the form of a programme that allows to choose various policies for resource allocation and their combination; to vary the amounts of budget intended for distribution in each stage of forecasting; to visually demonstrate distribution of resources in each forecasting period where the planned indicators are reached at the final moment of time in the optimal way. Optimality, in this case, is adherence to the objectives inherent in the objective functionals. 


\section{Results section}

Approbation of models of budget resources distribution was carried out on the basis of the municipal entity, Luzhsky municipal district. The documents of strategic planning served as the basis for the initial data: the Forecast of social and economic development of Luzhsky municipal district for 2018-2020, the Budget of the district, as well as technical and economic information provided by the Transport, Communications and Communal Services departments of the district [14] [15].

Luzhsky district is listed among the laggards of the Leningrad oblast in terms of attracting and absorbing investments. The situation is complicated due to the problems in the areas of creation of favorable conditions for investment, preparation of investment sites, and regulation of land relations.

Among the threats identified in the Luzhsky municipality, the following points are considered to be the most significant:

1. At present, it is relevant to involve new "green-field" land areas that have not been in demand among the individual investors before because of extremely high costs of infrastructure construction. However, the organizational and infrastructure preparation of investment sites in the region is weak, even for the implementation of strategically important investment regional projects.

2. The transport infrastructure within the district is insufficiently developed and the highways are in poor condition, which leads to the impossibility of using the entire area of the district for production location, as the access to certain parts of it is difficult.

3. The share of the able-bodied population in the district is decreasing, first, due to the natural decline, and second, due to the leakage of personnel to St. Petersburg. This is connected with the low level of the quality of life of the population, namely, the provision of housing, health care, education and culture.

4. The next threat is the discrepancy between the training of personnel and the needs of the economy. Even the enterprises operating in the region lack the necessary skilled personnel.

5. The low qualification of management creates difficulties in implementation of the latest management technologies. It is necessary to establish centers of retraining and upgrading of the skills of the middle and senior management.

In this regard, we proposed to adopt the following areas as priorities:

Transport infrastructure

1. Length of public roads with a hard surface

Engineering infrastructure

2. Length of heat and steam networks

3. Number of heating sources

4. Single mileage of a street water supply network

5. Single mileage of a street sewer network

6. Single mileage of street gas network

7. Cable electrical networks

Labour potential

8. Number of students in professional education institutions

9. Training and retraining of personnel

Quality of social environment

10. Housing deployment, total area

11. Provision of medical staff

12. Provision of medium-level medical personnel

13. Number of hospital beds

14. Number of cultural institutions 
The designed programme allowed to simulate various situations of development of the chosen areas depending on the amounts of budget assigned to distribution and choice of resource allocation policies. As a result of the performed calculations, we obtained the values of the monitored indicators of economic activity $\mathrm{y}_{i}(\mathrm{t})$ by years up to 2022. Moreover, at each moment of time we have the optimal distribution of a given amount $\mathrm{C}(\mathrm{t})$ as an $\mathrm{n}$ dimensional vector $u_{i}(t)$. Diagrams of the level of the monitored indicators and allocation of budget resources in the areas allow to visually observe the process of changing of the values of the monitored indicators. For the final decision on the choice of the resource allocation policy, the analysis of the results for all distribution options was carried out.

1. The model of "Equal direction development". As a result of application of this model, we observed the "pulling" of objects in proportion to their lag. Such distribution allows to transform simultaneously all objects from the initial state to the desired one (Fig. 1). The largest amount of budget resources is required for the development of the first direction, which is the development of transport infrastructure.

2. The model of "Distribution proportional to the required results" suggests that more budget resources are allocated to those areas where deviation from the standard is greater (Fig. 2). The greatest deviation from the standard is typical for areas nine ("Training and retraining of personnel") and ten ("Housing deployment"). Therefore, maximum amounts of budget are allocated to these areas. In general, this policy was not very successful because the first six areas associated with the development of engineering infrastructure are practically not developed.

3. The model of "Distribution proportional to the direction development level". When using this model, priority is given to the fast completion of work and achievement of a benchmark for areas with a high level of development. Therefore, in this case, the maximum amount of budget is allocated to the third, fourth, and fifth areas concerning the development of engineering infrastructure. However, the use of this distribution policy leads to the growth of disproportions in the development of areas. The model of "Distribution proportional to the direction development level"

4. The model of "Allowable deviations". When this model was implemented, allowable deviations from reference states were specified for individual areas. In particular, the allowable deviation for the areas of labour potential and the quality of the social situation is $p=0.2$. After reaching the normalized level of 0.8 , these areas are not invested anymore. All means go to the remaining areas. The model of "Allowable deviations"

5. The "Guaranteed minimum" model is used in case of vital, but underdeveloped areas, when there is a need for their rapid development at least to some extent. When using this policy, the following $q_{i}(t)$ values were set: $q_{1}(t)=0.1$ for the transport infrastructure development level, $q_{9}(t)=0.5$ for training, and $q_{10}(t)=0.3$ for housing deployment. In this situation, the normalized deviations of these areas from the standards in the next step after the distribution of budget should not exceed the specified values.

6. The "Priority setting" model. When using this model, specified priorities $e_{i}(t)$ are used as a criterion of priority instead of specific increments. In this case, it is possible to invest in the most important areas, which development is identified as a priority (Fig. 3). At each stage of the allocation, priorities can change. At the first stage, priority was given to areas six and nine, so a large amount of budget $e_{6}(t)=3, e_{9}(t)=3$ was allocated for their development. At the second stage, a need for a more rapid increase in the level of direction 14 was identified. At this stage, priority was set for this direction $e_{14}(t)=2$. At the third stage, the tenth direction $e_{10}(t)=3$ became a priority. At the fourth stage it was $e_{11}(t)=2.8$, and at the fifth it was $e_{9}(t)=2$.

As a result of the analysis, we can state that the following policies are the most reasonable: the model of "Equal direction development", "Guaranteed Minimum" and "Priority setting" models, which allow to vary the level of development of areas at 
intermediate stages depending on current priorities, and at the same time to approach the target values by the final forecasting period, aligning the disproportions of development.

This methodology can be used for a reasonable allocation of budget resources in priority areas for other municipalities.

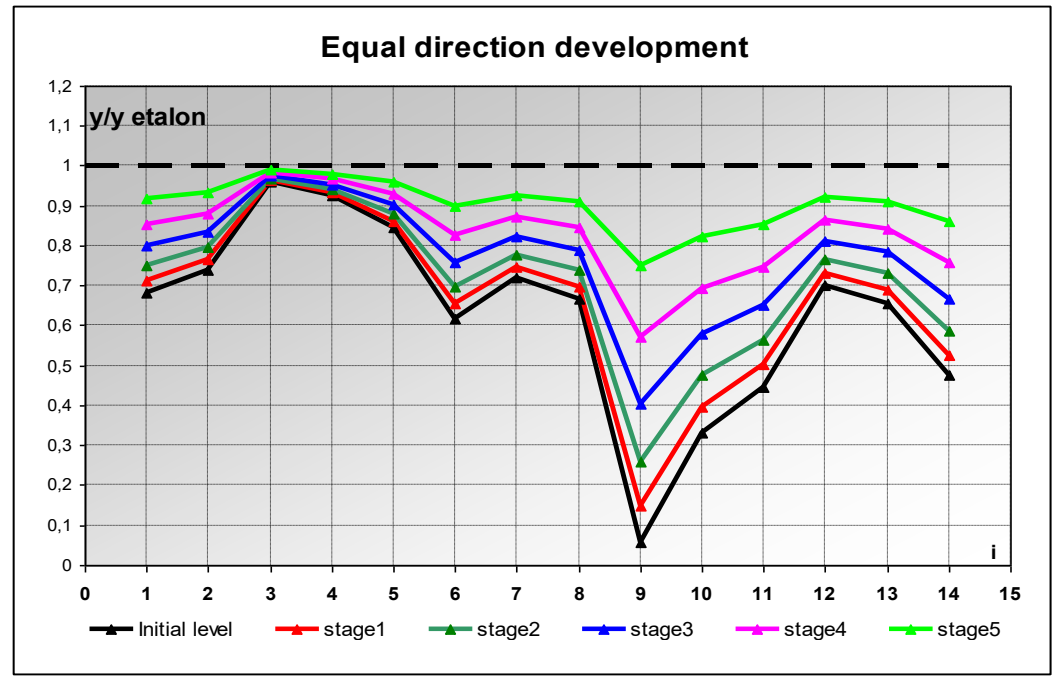

Fig. 1. The model "Equal direction development".

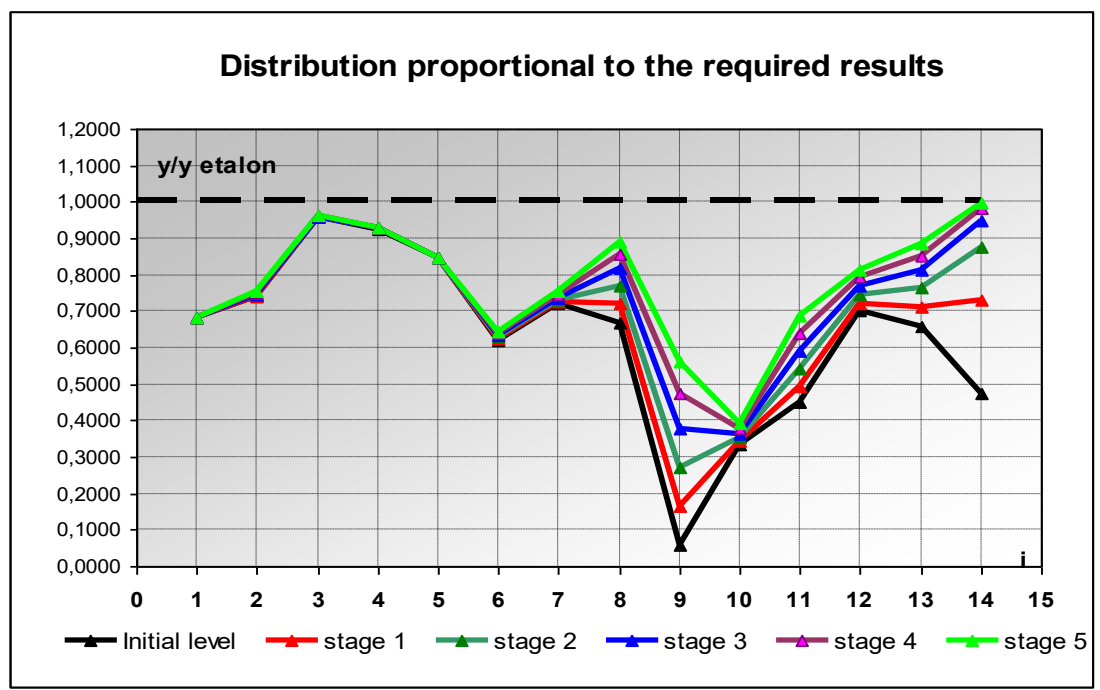

Fig. 2. The model "Distribution proportional to the required results". 


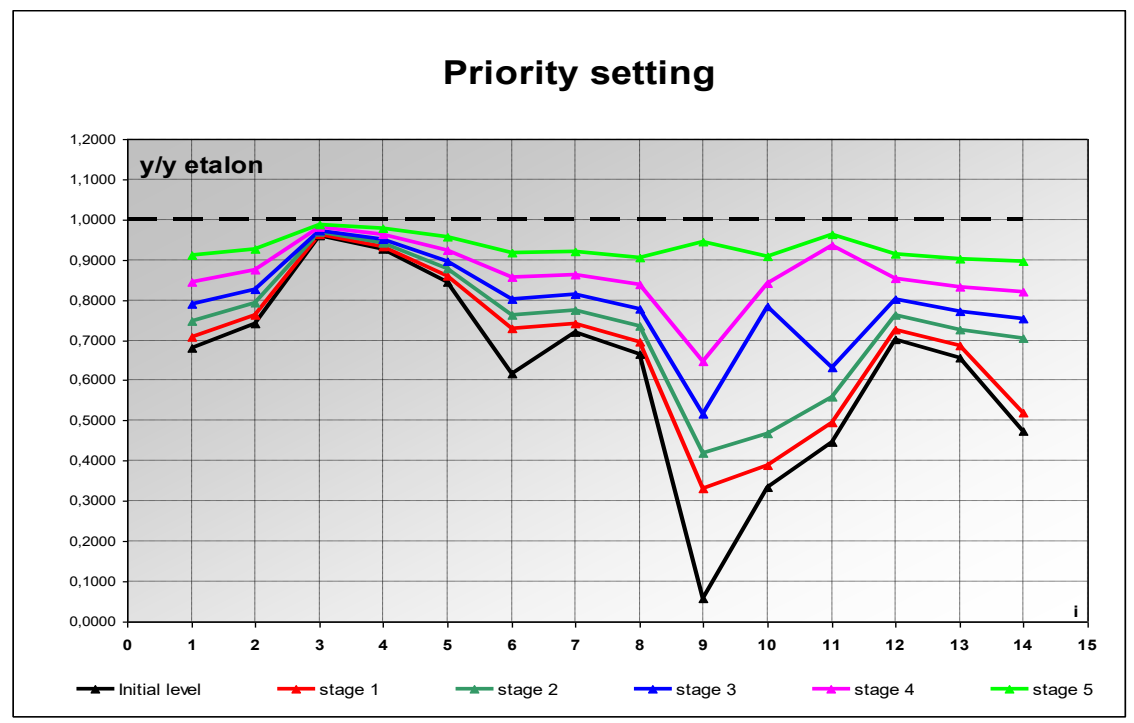

Fig. 3. The model "Priority setting".

\section{Discussion section}

In the development of decision-making models for allocation of costs, uncertainty arises from the ambiguity of expert estimates of standards, the amount of available resources in future periods, and the error in determining $s_{i}(t)$. The use of economic and mathematical models that do not take into account the influence of random factors can lead to a decrease in management efficiency. Consideration of the random factors impact is possible in stochastic models.

In addition, it is essential to take into account that the priorities in choosing the budget allocation policy will vary depending on the stage of the economic cycle.

In the recession phase, the main tasks are to smooth the impact of crisis consequences for the most affected areas and priority support to those areas that enable the system to pass the recession phase as soon as possible and prepare it for a rise in the period of the future recovery. If we choose a policy of equal distribution of budget resources at this stage, then the way out of the crisis will be longer and more complex.

Therefore, in the recession stage, the following policies are proposed as the most preferable:

1. The "Distribution proportional to the direction development level" policy with the purpose to give priority to areas with a high level of development.

2. The "Priority setting" policy with the given priorities $\mathrm{e}_{i}(\mathrm{t})$ as a criterion of priority instead of specific increments.

3. The "Guaranteed minimum" policy is applied in case of vital, but underdeveloped areas.

At the stage of recovery, when the basis for the most significant areas is formed by the task of regional policy, and there is a reduction of the imbalances in the development of remaining areas, the following policies are proposed as preferable:

1. The policy of "Equal direction development".is used in case when the main objective is the gradual smoothing of disproportions. 
2. "Distribution proportional to the direction development level" policy is relevant when a larger amount of budget is allocated to the areas with a greater level of deviation compared to the standard level.

3. The policy of "Allowable deviations". In the implementation of this model in areas that are underdeveloped or irrelevant, the allowable values of deviations are determined based on the benchmark states.

\section{Conclusions}

The designed model allows solving the task of forecasting the development of areas and the optimal allocation of resources at a given time interval with the selected planning interval. The choice of a certain policy is made by taking into account the peculiarities of municipal formation on the basis of analysis performed at the previous stages of the study. The number of areas considered in the model can be increased depending on the determined goals.

\section{References}

1. E.A. Kachanova, Municipality: Economics and Management http://municipal.uapa.ru/ru-ru/issue/2015/02/06/

2. A.P. Gradov, B.I. Kuzin, Mathematical models of the regional economy (St. Petersburg, 2002)

3. V. I. Korobko, Urban economy (Moscow, 2008)

4. A.V. Izotov, O.V. Rostova, Proceedings of the 29th International Business Information Management Association Conference, 1822-1832 (2017)

5. A.P. Gradov, Recurrence of economy development and competitive advantages management (St. Petersburg, 2011)

6. T.A. Khudyakova, A.V. Shmidt, Proceedings of the 12th International Conference on Strategic Management and its Support by Information Systems, 46-52 (2017)

7. S.V. Syatchikhin, A.G. Shelomentsev, Management matters 2, 240-248 (2017)

8. A.V. Ruchkin, S.A. Makovkina, Administrative consultation 8, 88-99 (2014)

9. V.B. Vasilyeva, Problems of modern economy 17, 48-51(2014)

10. A. Sen, The Idea of Justice (Moscow, 2004)

11. A.V. Shagov, V.V. Kolbin, Economics and Mathematical Methods 41, 54-64 (2015)

12. S.B. Suloeva, O.V. Rostova, Management of the investment process in the region (the concept, methods, tools) (St. Petersburg, 2009)

13. I.V. Ilin, A.V. Izotov, S.V. Shirokova, O.V. Rostova, A.I. Levina, SCM 20177519831 , 812-814 (2017)

14. Major indicators of the socio-economic development forecast of the Luga municipal district for 2018-2020, http://www.luga.ru/economika/pokaz/prognos

15. Budget of the Luga municipal district for 2018 and for the planning period 2019 and 2020, http://luga.ru/msu/adm/kom_otd/kom_fin/ispolneniebudjeta/reshenie/lmr 